\title{
AN OVERVIEW OF OSTEOMYELITIS WITH REFERENCE TO TREATMENT IN PARTICULAR MAGGOT DEBRIDEMENT THERAPY (MDT)
}

\author{
By \\ AHMED HASSAN FAWZI EL-TAWDY ${ }^{1}$, EISSA ABDEL HAMED IBRAHIM ${ }^{1}$, \\ AND TOSSON A. MORSY ${ }^{2}$
}

Military Medical Academy, Cairo $11291^{1}$ and Department of Parasitology, Faculty of Medicine, Ain Shams University, Cairo $11566^{2}$, Egypt

\section{Abstract}

Osteomyelitis occurs either as a result of hematogenous seeding, contiguous spread of infection to bone from adjacent soft tissues and joints, or direct inoculation of infection into the bone as a result of trauma or surgery. Hematogenous osteomyelitis is usually monomicrobial, while osteomyelitis due to contiguous spread or direct inoculation is usually polymicrobial. Staphylococcus aureus, coagulase-negative staphylococci and aerobic gram-negative bacilli are the most common organisms; other pathogens including streptococci, enterococci, anaerobes, fungi and mycobacteria have also been implicated. Acute osteomyelitis typically presents with gradual onset of pain over several days. Local findings (tenderness, warmth, erythema and swelling) and systemic symptoms (fever, rigors) may also be present. Chronic osteomyelitis tends to occur in the setting of previous osteomyelitis and presents with recurrent pain, erythema or swelling, sometimes in association with a draining sinus tract. Treatment of osteomyelitis often requires both surgical debridement of necrotic material and antimicrobial therapy for eradication of infection. The optimal duration of antibiotic therapy is not certain; but continuing parenteral antimicrobial therapy at least six weeks from the last debridement.

Maggot therapy is an effective and environmentally friendly treatment of complicated necrotic wounds that are resistant to conventional treatment and should also be considered in earlier stages of treatment. The history of maggot therapy, and the mechanisms by which it works, is discussed.

Key words: Osteomyelitis, microbial infection, Infection treatment, Maggot debridement therapy.

\section{Introduction}

Osteomyelitis is infection localized to bone (Berbari et al, 2005). It is one of the oldest re-corded diseases, with descriptions dating back to the time of Hippocrates (460$370 \mathrm{BC})$. The terms such as "abscessus in medulla," "necrosis", and "a boil of the bone marrow" were used to describe the infection until Nelaton introduced "osteomyelitis" in 1844. Prior to the introduction of penicillin in 1940, the management of acute osteomyelitis was purely surgical, with large incisions for removal of all necrotic bone (Lew and Waldvogel, 2004). Wounds were packed with vaseline gauze and left to heal by secondary intention after immobilization. Mortality rates remained high (about 33\%) due to sepsis until the introduction of penicillin that dramatically changed the treatment and prognosis of osteomyelitis. The complications such as the sequestration, sinus formation, and sepsis became less common and the goals of therapy changed from disease containment to cure.

Treatment: Osteomyelitis frequently requires both surgical therapy for debridement of the necrotic material together with anti-microbial therapy for eradication of infection. In certain circumstances, the surgical treatment may also require the hardware placement or removal and/or revascularization.

Post-traumatic osteomyelitis refers to the osteomyelitis that develops as a result of the contaminated open fractures or the surgical treatment of closed fractures. Post-traumatic osteomyelitis occurs in up to $25 \%$ of open fractures; risks depend upon the following factors (Lew and Waldvogel, 2004): Severity of fracture severity of soft tissue injury, bacterial contamination degree, the presence of underlying vascular insufficiency (e.g., peripheral vascular disease or diabetes).

The posttraumatic osteomyelitis pathogens may include skin flora, soil organisms, or 
nosocomial pathogens acquired in the hospital before, during, or after surgical intervention. Staphylococcus aureus, coagulasenegative staphylococci, and aerobic gramnegative bacilli are the commonest organisms; other pathogens, including the enterococci, anaerobes, fungi, and mycobacteria were implicated (Trampuz and Zimmerli, 2006).

The wound infections among combat casualties with tibial fractures have been associated with drug-resistant aerobic gramnegative organisms including Acinetobacter bauman-nii and Pseudomonas aeruginosa (Johnson et al, 2007). Cultures obtained at the time of initial debridement did not always reflect the pathogens involved once osteomyelitis has developed; the correlation was about 25\% (Kindsfater and Jonassen, 1995).

Clinically, Hallmarks of posttraumatic osteomyelitis are nonunion of the fracture site and poor wound healing after wound closure. Other symptoms may include fever and the local wound drainage, erythema, warmth, swelling, and pain. The tibia is the bone frequently involved by posttraumatic osteomyelitis, likely because it is the most common site of open fracture given its lack of muscle covering and limited anastomotic blood supply (Khatod et al, 2003).

Hematogenous osteomyelitis accounts for approximately $20 \%$ of cases of osteomyelitis in adults. For unclear reasons, it is more common in males regardless of age. Long bone and vertebral osteomyelitis are the two major types, but hematogenous osteomyelitis can occur in locations such as the pelvis and clavicle (Lew and Waldvogel, 2004). Patient characteristics that predispose to bacteremia, such as intravenous drug abuse, dialysis, and sickle cell disease, favor the development of the hematogenous osteomyelitis. Hematogenous osteomyelitis is primarily a disease of children, with $85 \%$ of cases occurring in patients younger than 17 years of age (Mader et al, 1999). However, the proportion of adult cases may be increasing as the mean age of the population rises in the United States and developed countries (Espersen et al, 1991). Most cases in adults are seen in patients over age 50, with the exception of intravenous drug users, the majority of who are under the age of 40 (Chandrasekar and Narula, 1986).

The hematogenous osteomyelitis is also the associated with other risk factors for the bacteremia (e.g., central lines, dialysis, urethral catheterization, urinary tract infection).

Treatment: The site of infection varies with age. In children, the most common sites of involvement are the long bones (e.g., femur, tibia, and humerus) with vertebral osteomyelitis accounting for only one to two percent of cases (Fernandez et al, 2000). In contrast, the long bones are rarely involved in adults; the major sites of infection are the vertebrae and the sternoclavicular and sacroiliac bones; the latter two most commonly occurring in intravenous drug users (Gordon and Lowy, 2005). Among adult patients with hematogenous infections of the hip, magnetic resonance imaging has demonstrated that infection commonly extended distal to the femoral head, into the medullary canal of the femur or acetabulum (Zalavras et al, 2009).

Among the patients with the vertebral osteomyelitis, the lumbar vertebral bodies are most often involved, followed in frequency by the thoracic and cervical vertebrae. The spread to adjacent vertebral bodies may occur rapidly through the rich venous networks in the spine. Posterior extension may lead to epidural and subdural abscesses or even meningitis. Extension anteriorly or laterally can lead to paravertebral, retropharyngeal, mediastinal, subphrenic, retroperitoneal, or psoas abscesses. Vertebral osteomyelitis was recognized as a distinct clinical entity by Hippocrates and Galen. Prior to the discovery of antibiotics, vertebral osteomyelitis was fatal in about $25 \%$ of affected patients (Kulowski, 1936).

Vertebral osteomyelitis is primarily a disease of adults, with the majority of 
patients being more than 50 years old. As a general rule, the age-adjusted incidence increases progressively with each successive decade of life. The men are affected approximately twice as often as women in most case series; the reason for this the male predominance was not clearly understood (Sapico and Montgomerie, 1979). It was difficult to obtain reliable information on the overall incidence of vertebral osteomyelitis. In two studies published in 1979 and 2001, the incidence of the spinal osteomyelitis was estimated to be 1:250,000 (Digby and Kersley, 1979) and 1:450,000, respectively (Beronius et al, 2001). However, most authorities believe that the overall incidence of vertebral osteomyelitis has steadily increased in recent years for three primary reasons: increasing rates of nosocomial bacteremia due to intravascular devices and other forms of instrumentation, increasing population age and injection drug use.

Treatment of infection associated with prosthetic joints is not standardized, because of the variable clinical presentations and the lack of data from randomized, controlled trials (Lentino, 2003). Treatment is difficult with initial success often followed by relapse once antibiotics are stopped, in part, due to biofilms sequestering the etiologic microorganism. The treatment should start immediately after the onset of the symptoms of infection to increase the probability of success. Treatment of the prosthetic joint infections usually involves the surgical debridement followed by a long course of intravenous antibiotics (Isiklar et al, 1994). The selection of the specific antimicrobial regimens depends upon the indentification infecting organism and is therefore critically dependent upon a definitive microbiologic diagnosis (Zimmerli et al, 1998). Two-stage replacement arthroplasty is associated with the highest success rates and therefore recommended in patients who are able to tolerate the long period of immobilization (Brandt et al, 1999). Arthrodesis (fusion of the joint) is the treatment of choice for patients with the poor skin coverage and inadequate tissue to provide the functional arthroplasty (Jorgensen and Torholm, 1995). The arthroscopic drainage and intravenous antimicrobial therapy followed by long-term suppression may be effective in up to $40 \%$ of patients with early-onset prosthetic knee infections and should be considered when patient was presented immediately after the onset of symptoms (Murdoch et al, 2001). Long-term suppressive therapy alone was sometimes necessary in patients who are elderly, have contra-indications to general anesthesia, or refuse to allow removal or debridement of an infected prosthesis (Berbari et al, 2006).

Antibiotic selection: The antibiotic therapy should be tailored to the culture and the sensitivity findings. If culture results are not obtainable, broad spectrum empiric therapy should be administered (Lazzarini et al, 2005).

For treatment of osteomyelitis due to the gram-negative organisms fluoroquinolones are excellent agents (if susceptibility testing confirms their sensitivity to the agent) since fluoroquinolones have high bone penetration, even with oral administration (Huddleston et al, 2000). Given the importance of biofilms in the pathophysiology of the staphylococcal osteomyelitis (particularly in the setting of hardware), some experts favor use of rifampin for activity against the microorganisms in biofilms, although others oppose its use given limited evidence for improved outcomes over the standard antimicrobial therapy. If used for treatment of osteomyelitis, rifampin must be combined with another active antibacterial agent due to the rapid emergence of resistance in the setting of rifampin monotherapy is common. Moreover, caution should be exercised regarding the risk of potential interactions between rifampin and other concurrently administered pharmaceutical agents (Henry et al, 1987).

Treatment of invasive methicillin-resistant $S$. aureus infection in adults: Methicillin re- 
sistance in $S$. aureus is defined as an oxacillin minimum inhibitory concentration (MIC) $\geq 4 \mathrm{mcg} / \mathrm{mL}$. Isolates resistant to oxacillin or methicillin were also resistant to all beta-lactam agents, including oxacillin, dicloxacillin, and cefazolin (Karchmer, 1991). Although the vancomycin largely remains the antibiotic of choice for the treatment of invasive methicillin-resistant Staphylococcus aureus (MRSA) infections, alternative agents must be considered in the setting of adverse effects to vancomycin or infection with a pathogen with inadequate susceptibility to vancomycin. In patients who fail to respond to vancomycin after seven days of therapy, the vancomycin MIC should be reassessed. Persistent bacteremia may be due to an endovascular source of infection that requires surgery. However, if antibiotic failure seemed to be the most likely explanation, if MIC approaches limit the susceptible range $(2 \mathrm{mcg} / \mathrm{ml})$, vancomycin must be discontinued and switched to an alternative antibiotic agent. If an alternative agent for treatment of MRSA bacteremia was needed due to vancomycin intolerance or inadequate response to treatment with daptomycin or linezolid (Grade 2C) and daptomycin to linezolid (Jenkins et al, 2008). The hospital-acquired (nosocomial) pneumonia (HAP), ventilator-associated pneumonia (VAP), and the health-care-associated pneumonia (HCAP) are important causes of the morbidity and mortality despite the improved antimicrobial therapy, supportive care, and prevention (CDC, 1994). Given the increasing prevalence of methicillin-resistant Staphylococcus aureus (MRSA) as both the nosocomial and the community-associated pathogen, beta-lactam antibiotics were no longer reliable empiric therapy for skin and soft tissue infections. Familiarity with local antibiotic patterns is important for selecting empiric antibiotic therapy, and culture with the susceptibility data was critical for tailoring treatment (Stevens et al, 2005). The recommended initial antibiotic management of MRSA osteomyelitis is with intravenous vancomycin (Grade 1C). If an alternative agent for treatment of MRSA osteomyelitis is needed due to vancomycin intolerance, and with daptomycin (Grade 2C)

Antibiotic therapy of the osteomyelitis requires a prolonged duration of treatment. This may be in part due to the observation in experimental models that $S$. aureus could persist following digestion by osteoblasts (Norden, 1988). In addition, the antibiotic penetration into the bone may be unreliable in some patients, particularly in those with the vasculopathy or prior extensive scarring from trauma. The optimal duration of the antibiotic therapy is not certain; the most experts favor the continuing parenteral antimicrobial therapy at least until debrided bone has been covered by vascularized soft tissue, which is usually at least six weeks from the last debridement.

Antimicrobial therapy may be administered on an outpatient basis via a long-term intravenous catheter with close monitoring; depending on antibiotic agent, monitoring may include weekly serum drug levels, renal function, liver function and/or hematologic function (Guglielmo et al, 2000).

Serial measurements of serum inflammatory markers (erythrocyte sedimentation rate and/or C-reactive protein) can be useful. If these test results have not normalized by the end of the planned treatment course, further clinical and/or radiographic investigation may be pursued. If orthopedic hardware that is known or presumed to be infected must be retained indefinitely, the long-term antibiotic suppression with an oral agent may be warranted. Such long term oral therapy is usually preceded by parenteral therapy until clinical signs of acute infection have resolved. Note that the duration of preceding parenteral therapy may be shorter in such cases than when definitive cure was the goal (Trampuz and Zimmerli, 2006).

If the osteomyelitic bone is fully resected (as when amputation is performed) a much shorter course of antibiotic therapy may be sufficient. In such cases, antibiotic therapy 
can usually be discontinued when operative wounds are healing without signs of the infection.

Adjunctive therapies for the osteomyelitis include hyperbaric oxygen (HBO) and vacuum-assisted closure. Osteomyelitis is often associated with reduced intraosseus blood flow and consequent reduced oxygen tension in infected bony tissue. This in turn might limit the neutrophil and macrophage activity (Chen et al, 2004). So, hyperbaric oxygen may be a useful adjunctive therapy in a small percentage of patients with refractory osteomyelitis. In 142 patients with refractory osteomyelitis treated with $\mathrm{HBO}$, successful healing without relapse was in $73 \%$ of them (Haas and McAndrew, 1996).

Hyperbaric oxygen (HBO) serves as primary or adjunctive therapy for a diverse range of medical conditions (show table 1(Gill and Bell, 2004). Most of the benefits of HBO are explained by the simple physical relationships determining gas concentration, volume, and pressure. The HBO is most commonly used under conditions of tissue hypoxia or to treat decompression sickness or gas embolism, in which gas bubbles obstruct blood flow. Henry's Law stated that the amount of an ideal gas dissolved in solution is directly proportional to its partial pressure. So, the dissolved plasma oxygen concentration of $0.3 \mathrm{~mL} / \mathrm{dL}$ at the sea level (1.0atm) increases to $1.5 \mathrm{~mL} / \mathrm{dL}$ upon the administration of $100 \%$ oxygen, while the hyperbaric oxygen delivered at 3.0atm yields a dissolved oxygen content of 6 $\mathrm{mL} / \mathrm{dL}$. The latter figure is sufficient to meet resting tissue oxygen extraction requirements irrespective of the adequacy of the hemoglobin-bound oxygen pool. The ability of HBO to augment oxygen content and independently meet resting tissue oxygen requirements has led to its use in conditions of compromised oxygen delivery, such as profound anemia, carbon monoxide $(\mathrm{CO})$ poisoning, and both acute and chronic ischemia (Van Meter, 2005). Generally, the $\mathrm{HBO}$ is recommended in severe decom- pression sickness or arterial gas embolism, some cases of carbon monoxide poisoning. Carbon monoxide (CO) is an odorless, tasteless, colorless, nonirritating gas formed by hydrocarbon combustion. Atmospheric concentration of $\mathrm{CO}$ is generally below $0.001 \%$, but it may be higher in urban areas or enclosed environments. CO binds to the hemoglobin with much greater affinity than the oxygen, forming carboxyhemoglobin $(\mathrm{COHb})$ and resulting in impaired oxygen transport and utilization. The $\mathrm{CO}$ can also precipitate an inflammatory cascade that results in CNS lipid peroxidation and delayed neurologic sequelae and overall case-fatality rate for $\mathrm{CO}$ poisoning ranges from 0 to 31\% (Hampson and Hauff, 2008). Smoke inhalation, carbon monoxide (CO) poisoning, $\mathrm{CO}$ is an odorless, tasteless, colorless, nonirritating gas formed by the incomplete combustion of carbon-containing compounds (Ernst and Zibrak, 1998). The clinical findings of $\mathrm{CO}$ toxicity are highly variable and largely nonspecific. Symptoms and signs may include headache, nausea, malaise, altered cognition, dyspnea, angina, seizures, cardiac dysrhyth-mias, congestive heart failure, and/or coma (Alarie, 2002).

HBO may be useful in the care of patients with cyanide poisoning, severe anemia, actinomycotic brain abscesses, acute crush injuries, prior radiation therapy, aggressive soft tissue infections, non-healing ulcers, or compromised skin grafts and flaps. Further research is required in these situations in order to confirm the benefits of the HBO and justify its significant costs and potential risks.

Vacuum assisted wound closure (VAC, also called negative pressure wound therapy) is an adjunctive therapy in treatment of open wounds that follows surgical debridement. Based on observation that controlled, the subatmospheric pressure applied to an open wound accelerated the healing and closure (Argenta and Morykwas, 1997). The VAC becomes the preferred treatment modality for many complex or chronic wounds. 
Examples of VAC systems include the vacuum assisted closure $\left(\mathrm{VAC}^{\circledR}\right)$ device (KCI, San-Antonio, Texas, USA) and the Chariker-Jeter ${ }^{\circledR}$ wound sealing kit (Smith and Nephew PLC, London, UK). But, the contraindications to VAC included the incomplete debridement, a visible vascular structure in the wound, malignancy in the wound, untreated osteomyelitis, or an open fistula. Necrotic tissue should be debrided from the wound before application. Wound dead tissue exacerbates inflammation and serves as a focus of infection. Bleeding can occur when the sponge contacts a vascular structure (White et al, 2005). Also, the malignancy within the wound, osteomyelitis not debrided and treated with appropriate antibiotic therapy and open fistulas to organs or body cavities (Whelan et al, 2005).

Occasionally acute osteomyelitis can be associated with disabling or life threatening complications in the absence of appropriate therapy. Suppurative infection may involve contiguous structures such as the joints and soft tissues, leading to sinus tract formation. Osteolysis and pathologic fractures were described, although such complications are exceedingly rare with early recognition and treatment. Infrequently, the hematogenous spread and sepsis could occur, although it might be difficult to determine whether the primary source of infection was the bloodstream or bone (Gelfand et al, 2006).

Sinus tract formation may be associated with neoplasms, especially in the setting of longstanding infection range 4 to 50 years (Altay et al, 2004). The squamous cell carcinoma (SCC) is the commonest tumor associated with chronic osteomyelitis; other authors reported that tumors were fibrosarcoma, myeloma, lymphoma, plasmacytoma, angiosarcoma, rhabdomyosarcoma, and malignant fibrous histiocytoma (McGrory et al, 1999). Most patients with neoplasm have a history of repeated surgical interventions. The development of a malignant tumor is heralded by an enlarging mass, increasing pain, foul-smelling drainage, bleeding, or radiographic evidence of bone destruction. Therefore, recalcitrant infection that does not respond to conventional therapy should prompt biopsy evaluation for malignancy from multiple sites including the ulcer, sinus tract and underlying bone.

What is maggot therapy?

Maggot therapy is the controlled, therapeutic use of live blow fly larvae "maggots" to treat skin and soft tissue wounds. The history of maggot therapy, and mechanisms by which it works, will be discussed below. But first, some disclosures.

Do maggots clean wounds? It is a type of biotherapy involving the introduction of live, disinfected maggots (fly larvae) into non-healing skin and soft tissue wound(s) of a human or animal for the purpose of cleaning out the necrotic (dead) tissue within a wound (debridement) and disinfection.

Baer (1918) during the Great War, served as a consulting physician with the American Expeditionary Force in France. Baer treated two soldiers who had endured their injuries on the battlefield for a week before being brought to a military hospital. He noted the lack of any systematic infection, fever, or purulence and that the wounds displayed the "most beautiful pink granulation tissue that one can imagine. Baer (1931) in USA had successfully used maggots in osteomyelitis treatment in four children in the 1930'. After many successes in the 1930' the maggots therapy became limited to the intractable wounds after introduction of sulphonamides and mass-production of the Flemming's penicillin. The present use of maggots came in the 1980' when the better methods of sterilization of both eggs and maggots were developed and clinical efficiency of the antibiotics used for the wound treatment dramatically decreased. Today the maggots' therapy became less treatment of last resort but of first choice in leg ulcers, carbuncules, pressure ulcers and the infected traumatic wounds especially in diabetic foot and in destroying malignant tissue as well. Easiness in application, safety, without side effects 
and often exceptional efficiency in wound debridement makes maggots therapy the first line therapeutic tool in both hospital and outpatient surgery. From Clinical experience they concluded that maggot therapy might reduce costs of treatment considerably by shortening hospital stay and decrease usage of antibiotics. McKeever (1933) credited Baer (1931) with the development of the approach in osteomyelitis based on the observations Baer made in France during the First World War (WWI). Baer noted that the previous writers (including the great French war surgeon and surgeon-in-chief to $\mathrm{Na}$ poleon's Armies, Dominique Jean Larrey) had mentioned the beneficial effects of the maggots in the soft tissue wounds throughout the 1800s. Thus, the concept was wellknown, if not widely used, and not specifically applied to the chronic osteomyelitis. McKeever described in detail the production of non-sterile maggots and how to apply them in chronic osteomyelitis. While the beneficial effects of maggots in wounds and in osteomyelitis in particular have been documented over the years, they have never gained widespread use. Nonetheless, the United States Food and Drug Administration approved the use of sterile maggots (FDA, 2004). Zumpt (1065) in Germany described myiasis as "the infestation of live human and vertebrate animals with dipterous larvae, which at least for a period, feed on the host's dead or living tissue, liquid body substances, or ingested food". For modern purposes however, this was too vague. For example, feeding on dead or necrotic tissue was not generally a problem except when larvae such as those of flies in family Piophilidae attack stored food such as cheese or preserved meats; such activity suggested saprophagy rather than parasitism; it even may be medically beneficial in maggot debridement therapy (MDT).

Sherman and Pechter (1988) in USA employed the larvae of some members of Diptera: Calliphoridae, notably the Lucilia illustris (Meigen), L. sericata (Meigen) and
Phormia regina (Meigen) for the maggot therapy, i.e. to help in cleaning the lesions antiseptically, especially for the treatment of chronic osteomyelitis. These maggots mode of treatment remained appropriate for the patients where antibiotics were ineffective and surgery impracticable.

Mumcuoglu et al. (1997) in Jerusalem treated five patients with diabetic-foot by the maggot therapy. The most serious case was in a 75-year-old man with gangrene and osteomyelitis of the right foot. The Proteus mirabilis, Enterococcus sp., Providencia stuartii and Staphylococcus spec. (coagulase positive) were isolated from lesions that did not respond to antibiotic therapy. The patient had twice refused amputation but agreed to the maggot therapy. Larvae of L. sericata were used for twice-weekly treatment over a period of seven months. Sterile larvae were applied to the wound and replaced every 3-4 days. After four months of treatment, the necrotic tissue around the toes and on the sole of the foot detached from the healthy tissue. During the last 3 months of treatment the larvae removed the remaining infected tissue. As therapy progressed, new layers of healthy tissue covered the wound. The offensive odor associated with the necrotic tissue and the intense pain in the patient foot decreased significantly. At the end of the maggot therapy, during which there were no complaints of discomfort, the patient was able to walk. In the other four patients who had relatively superficial gangrene, maggots debrided the wounds within 2-4 weeks. They recommended maggot therapy in cases of the intractable gangrene and osteomyelitis, when treatment with antibiotics and surgical debridement failed. Galeano et al. (2001) stated that the maggot therapy for treatment of osteomyelitis and deep wounds simple and inexpensive, effective and readily be used worldwide, particularly when the antibiotics were not available and/or ineffective and when surgery was impractical.

Rayner (1999) in Chelsea and Westminster Hospital, London reported that the use of 
larvae (maggots) in wound management was popular in the 1930s. Now the advent of multi-resistant strains of bacteria led to its reintroduction in some hospitals, when other avenues have been exhausted.

Steenvoorde and Jukema (2004) in The Netherlands reported that the number of maggots needed to debride a wound was estimated to be at $10 / \mathrm{cm}^{2}$ and more in case of a higher percentage of necrosis or slough. In their own hospital, from March 1999 to May 2002, successfully treated sixteen osteomyelitis patients with trauma as the etiological factor with the maggot therapy; average time was 27 days, with an average of seven maggot changes. In-vitro findings showed that maggot therapy was more effective in gram-positive infected wounds $(p=0.001)$. The opposite effect was found for the gram-positive infected wounds (nonsignificant $\mathrm{p}=0.07)$. In vivo the maggots therapy were less effective against the gramnegative infected wounds. They believed that a higher number of maggots needed not only for a larger wound or a wound with a higher percentage covered with slough, but also for a wound infected with gramnegative bacteria.

Jukema et al. (2006) in The Netherlands stated that the ancient method of larval therapy for treatment of acute and chronic infections has become a revival and a new dimension with introduction of the Biobag (Vitapad). They concluded that the trauma patients suffering infectious complications could be treated very effective, with reduce in overall time needed for the treatment and diminished invalidity.

Orkiszewski (2007) in Poland stated that although a beneficial effect of the wound infestation with maggots was known for many centuries, but not until dr. Zacharias recognized medical importance of maggots during the American Civil War. He intentionally introduced the maggots into the wound for its debridement.

Falch et al. (2009) in Norway reported that maggot therapy of the medical use of the disinfected fly larvae of $L$. sericata in the treatment of wounds resistant to the conventional treatment. They added that the maggots work through three mechanisms of action; they debride wounds by dissolving necrotic tissue, clean wounds by killing bacteria and promote wound healing. The larvae have a broad antibacterial action against Gram-negative and Gram-positive bacteria, including MRSA. Maggot therapy is used to debride a number of complicated skin and soft tissue wounds - e.g., pressure ulcers, venous stasis ulcers, neurovascular ulcers, traumatic wounds and burns - but also as a treatment for osteomyelitis. Large controlled clinical trials have not yet performed. Maggot therapy was associated with serious side effects.

Rozin et al. (2011) in Israel pose a therapeutic challenge and often resistant to treatment were treated by maggots therapy. Case 1: A 55-year old woman presented with long-standing painful LLU due to mixed connective tissue disease (MCTD). Biopsy from the ulcer edge showed small vessel vasculitis. The IV methylprednisolone (MethP) 1G/day, prednisolone (PR) 1mg/kg, monthly IV cyclophosphamide (CYC), cyclosporine (CyA) 100mg/day, IVIG 125G, ciprofloxacin+IVIloprost+enoxaparin+aspiri n (AAVAA), hyperbaric oxygen therapy (HO), maggot debridement and autologous skin transplantation were performed and the LLU healed. Case 2: A 45-year old women with MCTD developed multiple LLU's with the non-specific inflammation by biopsy. MethP, PR, hydroxychloroquine (HCQ), azathioprine (AZA), CYC, IVIG, AAVAA failed. Treatment for underlying the LLU tibial osteomyelitis and addition of CyA was followed by the LLU healing. Case 3: A 20year-old man with history of polyarteritis nodosa (PAN) developed painful LLU's due to small vessel vasculitis (biopsy). MethP, PR $1 \mathrm{mg} / \mathrm{kg}$, CYC, CyA $100 \mathrm{mg} / \mathrm{d}$, AAVAA failed. The MRSA sepsis and relapse of systemic PAN developed. IV vancomycin, followed by ciprofloxacin, monthly IVIG 
$(150 \mathrm{~g} /$ for 5 days) and infliximab $(5 \mathrm{mg} / \mathrm{kg})$ were instituted and the LLU's healed.

Marineau et al. (2011) in Hawaii treated 23 diabetic patients with MDT. They concluded that maggots were able to debride the diabetic wounds and stimulate wound healing, and demonstrated that MDT proved to be an effective strategy for the treatment of complex, diabetic wounds. Furthermore, the authors have shown that MDT works in dry, gangrenous wounds as well. Patient acceptance of, and satisfaction with, MDT was excellent. The majority of the patients tolerated the MDT well with only a few experiencing pain that was adequately controlled with oral analgesics. Vijay et al. (2013) in USA reported a case of furuncular myiasis in an immigrant from El Salvador with magnetic resonance imaging findings, which was unique because neuroimaging was obtained upon the clinical suspicion of calvarial osteomyelitis.

Choudhary et al. (2016) reported that the maggot debridement therapy (MDT) is a safe, effective, and controlled method of healing of chronic wounds by debridement and disinfection. In this therapy live, sterile maggots of green bottle fly, L. (Phaenicia) sericata were used, as they preferred the necrotic tissues over healthy for feeding. Since centuries, MDT is used in human beings to treat the chronic wounds. Lately, MDT came out as a potent medical aid in animals. In animals, although, this therapy is still limited and clinical studies are few. However, with the increasing antibiotic resistance and chronic wound infections in veterinary medicine, maggot therapy may even become the first line of treatment for some infections. MDT and its role in veterinary medicine add one more treatment method to utilize in non-healing wounds of animals and overcome the use of amputation and euthanasia. They concluded that MDT is frequently used in human medicine although this therapy is still limited in animals and clinical studies are few. The maggots are supplied by licensed laboratories in sterile bottles, which make it very safe, efficient, and easy method of healing for chronic wounds and prevents secondary bacterial infections. There is an overwhelming need for improved wound care in countries which are under-provided for medical veterinary facilities. In veterinary medicine, further clinical studies are needed in several fields, including establishing a number of maggots required for safe and efficient treatment and identification of the adverse effect during treatment. El Tawdy et al. (2015) critically reviewed the MDT and its' safe use for treating a diabetic foot ulcer.

\section{Summary and Recommendations}

Osteomyelitis occurs either as a result of hematogenous seeding, contiguous spread of infection to bone from adjacent soft tissues and joints, or direct inoculation of infection into bone as a result of trauma or surgery. Hematogenous osteomyelitis is usually monomicrobial, while osteomyelitis due to contiguous spread or direct inoculation is usually polymicrobial. The Staphylococcus aureus, coagulase-negative staphylococci and aerobic gram-negative bacilli are the most common organisms; other pathogens including streptococci, enterococci, anaerobes, fungi and mycobacteria are implicated. Acute osteomyelitis typically presents with gradual onset of pain over several days. Local findings (tenderness, warmth, erythema and swelling) and systemic symptoms (fever, rigors) may also be present. Chronic osteomyelitis tends to occur in the setting of previous osteomyelitis and presents with recurrent pain, erythema or swelling, sometimes in association with a draining sinus tract. Most cases of diabetic foot osteomyelitis occur in the setting of ulcers that develop in absence of exposed bone. If a diabetic foot ulcer is larger than $2 \times 2 \mathrm{~cm}$ or bone is palpable, osteomyelitis is so likely that additional noninvasive the evaluation may not be needed. Positive results of a probe-to-bone test are sufficient for osteomyelitis diagnosis. Establishing an accurate diagnosis is critical, since infection can lead 
to prolonged antibiotic therapy and/or the aggressive surgical intervention. Reference standard for diagnosis of osteomyelitis is isolation of bacteria from a bone biopsy sample obtained via sterile technique, together with the histologic findings of inflammation and osteonecrosis. The bone biopsy may not be needed for patients with radiologic studies consistent with the osteomyelitis in the setting of positive blood cultures. Cultures obtained from sinus tract drainage are not reliable. Open biopsy is preferable over needle biopsy; bone samples should be obtained at the time of surgical debridement if performed. Percutaneous needle biopsy is an alternative to open the biopsy; ideally it should be performed via the intact tissues and under radiographic guidance to optimize yield. The bone biopsy should be obtained prior to treatment with antimicrobial therapy whenever possible. Leukocytosis may be observed in the setting of acute osteomyelitis but is less common in the setting of chronic osteomyelitis. The erythrocyte sedimentation rate (ESR) and/or C-reactive protein (CRP) are usually elevated but may be normal. Blood cultures are most likely to be positive in the setting of hematogenous infection and vertebral disease; positive blood cultures may obviate the need for invasive diagnostic testing. The treatment of osteomyelitis often requires both the surgical debridement of necrotic material and the antimicrobial therapy for infection eradication. The optimal duration of antibiotic therapy is not certain; thus continuing parenteral antimicrobial therapy at least six weeks from the last debridement was suggested (Grade 2B).

Undoubtedly, the maggots' ability to prevent the infections and promote wound healing is known since the $19^{\text {th }}$ Century. The increasing problems with treatment-resistant wounds and antibiotic-resistant bacteria have aroused interest in maggot therapy. There are numerous dressings to choose from, with costly new products coming to the market on a monthly basis, all claiming to improve the outcomes. The maggot debridement therapy (MDT) has been infrequently used due to improve the dressings, the new surgical techniques, and the surge of new antibiotics to treat the nonhealing wounds when they become infected. The medical-grade maggots became commercially available in 2004, and today there is a resurgence of interest in the MDT with 12 laboratories in 20 countries dispensing them at low cost. They are approved for debridement of necrotic tissue wounds, including pressure ulcers, venous ulcers, neuropathic foot ulcers, and non-healing traumatic or the post-surgical wounds (International Diabetes Federation, 2010).

\section{References}

Alarie, Y, 2002: Toxicity of fire smoke. Crit. Rev. Toxicol. 32:259-64.

Altay, M, Arikan, M, Yildiz, Y, Saglik, Y, 2004: Squamous cell carcinoma arising in chronic osteomyelitis in foot and ankle. Foot Ankle Int. 25:805-9.

Argenta, LC, Morykwas, MJ, 1997: Vacuumassisted closure: A new method for wound control and treatment: Clinical experience. Ann. Plast. Surg. 38:563-9.

Baer, WS, 1918: Symposium on Arthroplasty: Arthroplasty with the aid of animal membrane. J. Orthop. Surg. 16:1-29.

Baer, WS, 1931: The treatment of chronic osteomyelitis with the maggot (larva of the blow fly) J. Bone Joint Surg. Am. 13:438-75

Berbari, EF, Osmon, DR, Duffy, MC, et al, 2006: Outcome of prosthetic joint infection in patients with rheumatoid arthritis: the impact of medical and surgical therapy in 200 episodes. Clin. Infect. Dis. 42:216-22

Berbari, EF, Steckelberg, JM, Osmon, DR, 2005: Osteomyelitis. In: Principles and Practice of Infectious Diseases, $6^{\text {th }}$ ed, Mandell, GL et al (eds), Elsevier, Philadelphia.

Beronius, M, Bergman, B, Andersson, R, 2001: Vertebral osteomyelitis in Goteborg, Sweden: A retrospective study of patients during 1990-95. Scand. J. Infect. Dis. 33:527-32.

Brandt, CM, Duffy, MCT, Berbari, EF, et al, 1999: Staphylococcus aureus prosthetic joint infection treated with prosthesis removed and delayed reimplantation arthroplasty. Mayo Clin. Proc. 74:553-9. 
CDC, 1994: Guideline for prevention of nosocomial pneumonia. Respir Care 39:1191-9.

Chandrasekar, PH, Narula, AP, 1986: Bone and joint infections in intravenous drug abusers. Rev. Infect. Dis. 8:904-9.

Chen, CE, Ko, JY, Fu, TH, Wang, CJ, 2004: Results of chronic osteomyelitis of the femur treated with hyperbaric oxygen: A preliminary report. Chang Gung Med. J. 27:91.

Choudhary, V, Choudhary, M, Pandey, S, Hasnani, JJ, 2016: The maggot debridement therapy as primary tool to treat chronic wound of animals. Vet. World. 9, 4): 403-9. Published online 2016 Apr 25.

Cierny, G, Mader, JT, Pennick, H, 1985: Contempt Orthopdic 10:17-22.

Digby, JM, Kersley, JB, 1979: Pyogenic nontuberculous spinal infection: an analysis of thirty cases. J. Bone Joint Surg. Br. 61:47-52.

El-Tawdy, AH, Ibrahim, EA, Abdallah, ES, Al Sakhawy, EM, Morsy, TA, 2016: Maggot debridement therapy (MDT): it is safe and economic for treating a diabetic foot ulcer. J. Egypt. Soc. Parasitol. 46, 1:223-34.

Ernst, A, Zibrak, JD, 1998: Carbon monoxide poisoning. N. Engl. J. Med. 339:1603-6.

Espersen, F，Frimodt-Moller，N，Thandrup Rosdahl, V, et al, 1991: Changing pattern of bone and joint infections due to Staphylococcus aureus: Study of cases of bacteremia in Denmark: 1959-1988. Rev. Infect. Dis. 13:347-52.

Falch, BM, de Weerd, L, Sundsfjord, A, 2009: Maggot therapy in wound management. Tidsskr Nor Laegeforen. 129, 18:1864-7.

FDA, 2004: Food and Drug Admionstration Website. Available at: http://www.fda.gov/cdrh/ 510k/sumjan04.html. Accessed March 11, 2008.

Fernandez, M, Carrol, CL, Baker, C, 2000: Discitis and vertebral osteomyelitis in children: an 18-year review. Pediatrics 105:1299-302.

Galeano, M, Ioli, V, Colonna, M, Risitano, G, 2001: Maggot therapy for treatment of the osteomyelitis and deep wounds: an old remedy for an actual problem. Plast. Reconstr. Surg. 108:2178-9.

Gelfand, MS, Cleveland, KO, Heck RK, Goswami, R, 2006: Pathological fracture in acute osteomyelitis of long bones secondary to community-acquired methicillin-resistant Staphylococcus aureus: Two cases and review of the literature. Am. J. Med. Sci. 332:357-62.

Gill, AL, Bell, CN, 2004: Hyperbaric oxygen: its uses, mechanisms of action and outcomes. QJM 97:385-92.

Gordon, RJ, Lowy, FD, 2005: Bacterial infections in drug users. N. Engl. J. Med. 353: 1945-52.

Guglielmo, BJ, Luber, AD, Paletta, D Jr, Jacobs, RA, 2000: Ceftriaxone therapy for staphylococcal osteomyelitis: A review. Clin. Infect. Dis. 30:205-9.

Haas, DW, McAndrew, MP, 1996: Bacterial osteomyelitis in adults: evolving considerations in diagnosis and treatment. Am. J. Med.101:5505.

Hampson, NB, Hauff, NM, 2008: Risk factors for short-term mortality from carbon monoxide poisoning treated with hyperbaric oxygen. Crit. Care Med. 36:2523-8.

Huddleston, PM, Steckelberg, JM, Hanssen, AD, et al, 2000: Ciprofloxacin inhibition of experimental fracture healing. J. Bone Joint Surg. Am. 82:161-8.

International Diabetes Federation, 2010: Position statement, The diabetic foot. Available at: http://www.idf.org/position-statement-diabetic foot.

Isiklar, ZU, Landon, GC, Tullos, HS, 1994: Amputation after failed total knee arthroplasty. Clin. Orthop. 299:173-8.

Jenkins, TC, Price, CS, Sabel, AL, et al, 2008: Impact of routine infectious diseases service consultation on the evaluation, management, and outcomes of Staphylococcus aureus bacteremia. Clin. Infect. Dis. 46:1000-12.

Johnson, EN, Burns, TC, Hayda, RA, et al, 2007: Infectious complications of open type III tibial fractures among combat casualties. Clin. Infect. Dis. 45:409-11.

Jorgensen, PS, Torholm, C, 1995: Arthrodesis after infected knee arthroplasty using the long arthrodesis nail: A report of five cases. Am. J. Knee Surg. 8:110-9.

Jukema, GN, Steenvoorde, P, Wong, CY, Bernards, AT, van Dissel, JT, 2006: Maggot therapy for treatment of severe infections in trauma surgery: "back to the future!". Zentralbl. Chir. 131, 1:S75-8.

Karchmer, AW, 1991: Staphylococcus aureus and vancomycin: The sequel. Ann. Int. Med. 115:739-42.

Khatod, M, Botte, MJ, Hoyt, DB, et al, 2003: Outcomes in open tibia fractures: relationship between delay in treatment and infection. J. Trauma 55:949-52. 
Kindsfater, K, Jonassen, EA, 1995: Osteomyelitis in grade II and III open tibia fractures with late debridement. J. Orthop. Trauma 9:121-8.

Kulowski, J, 1936: Pyogenic vertebral osteomyelitis of the spine: An analysis and discussion of 102 cases. J. Bone Joint Surg. 1:343-6.

Lazzarini, L, Lipsky, BA, Mader, JT, 2005: Antibiotic treatment of osteomyelitis: what have we learned from 30 years of clinical trials? Int. J. Infect. Dis. 9:127-32.

Lentino, JR, 2003: Prosthetic joint infections: bane of orthopedists, challenge for infectious disease specialists. Clin. Infect. Dis. 36:1157-60. Lew, DP, Waldvogel, FA, 1997: Osteomyelitis. N. Engl. J. Med. 336:999-1004.

Lew, DP, Waldvogel, FA, 2004: Osteomyelitis. Lancet; 364:369-72.

Mader, JT, Shirtliff, M, Calhoun, JH, 1999: The host and the skeletal infection: classification and pathogenesis of acute bacterial bone and joint sepsis. Baillieres Best. Pract. Res. Clin. Rheumatol. 13:1-9.

Marineau, ML, Herrington, MT, Swenor, K M, Eron, LJ, 2011: The maggot debridement therapy in the treatment of complex diabetic wounds. Hawaii Med. J. 70, 6:121-4.

McGrory, JE, Pritchard, DJ, Unni, KK, et al, 1999: Malignant lesions arising in chronic osteomyelitis. Clin. Orthop. Relat. Res.12:181-8.

McKeever, DC, 1933: Maggots in treatment of osteomyelitis: a simple inexpensive method. J. Bone Joint Surg. Am. 15:85-93.

Mumcuoglu, KY, Lipo, M, Ioffe-Uspensky, I, Miller, J, Galun, R, 1997: Maggot therapy for gangrene and osteomyelitis. Harefuah 132, 5: $323-5,-82$.

Murdoch, DR, Roberts, SA, Fowler, VG Jr, et al, 2001: Infection of orthopedic prostheses after Staphylococcus aureus bacteremia. Clin. Infect. Dis. 32:647-54.

Norden, CW, 1988: Lessons learned from the animal models of osteomyelitis. Rev. Infect. Dis. 10:103-9.

Orkiszewski, M, 2007: Maggots of Lucilia sericata in treatment of intractable wounds. Wiad. Lek. 60, 7/8:381-5.

Rayner, K, 1999: Larval therapy in wound debridement. Prof. Nurse 14, 5:329-33.

Rozin, AP, Egozi, D, Ramon, Y, Toledano, K, Braun-Moscovici, Y, et al, 2011: Large leg ulcers due to autoimmune diseases. Med. Sci. Monit. 17, 1:CS1-7.
Sapico, FL, Montgomerie, JZ, 1979: Pyogenic vertebral osteomyelitis: Report of nine cases and review of the literature. Rev. Infect. Dis. 1:7549.

Sherman, RA, Pechter, EA, 1988: Maggot therapy: A review of the therapeutic applications of fly larvae in human medicine, especially for treating osteomyelitis. Med. Vet. Entomol. 2, 3: 225-30.

Sherman, RA, Pechter, EA, 1988: Maggot therapy: A review of the therapeutic applications of fly larvae in human medicine, especially for treating osteomyelitis. Med. Vet. Entomol. 2:22530 .

Steenvoorde, P, Jukema, GN, 2004: The antimicrobial activity of maggots: in-vivo results. J. Tissue Viability 14, 3:97-101.

Stevens, DL, Bisno, AL, Chambers, HF, et al, 2005: Practice guidelines for the diagnosis and management of skin and soft-tissue infections. Clin. Infect. Dis. 41:1373-80.

Trampuz, A, Zimmerli, W, 2006: Diagnosis and treatment of infections associated with fracture-fixation devices. Injury 37:2:S59-62.

Trampuz, A, Zimmerli, W, 2006: Diagnosis and treatment of the infections associated with fracture-fixation devices. Injury 37, 2:S59-64.

Van Meter, KW, 2005: A systematic review of the application of hyperbaric oxygen in the treatment of severe anemia: an evidence-based approach. Undersea Hyperb. Med. 32:61-9.

Vijay, K, Kalapos, P, Makkar, A, Engbrecht, B, Agarwal, A, 2013: Human botfly (Dermatobia hominis) larva in a child's scalp mimicking osteomyelitis. Emerg. Radiol. 20, 1:81-3.

Whelan, C, Stewart, J, Schwartz, BF, 2005: Mechanics of wound healing and importance of vacuum assisted closure in urology. J. Urol. 173: 1463-9.

White, RA, Miki, RA, Kazmier, P, Anglen, J O, 2005: Vacuum-assisted closure complicated by erosion and hemorrhage of the anterior tibial artery. J. Orthop. Trauma 19:56-61.

Zalavras, CG, Rigopoulos, N, Lee, J, et al, 2009: Magnetic resonance imaging findings in hematogenous osteomyelitis of the hip in adults. Clin. Orthop. Relat. Res. 467:1688-94.

Zimmerli, W, Widmer, AF, Blatter, M, et al, 1998: Role of rifampin for treatment of orthopedic implant-related staphylococcal infections. JAMA 279:1537-44.

Zumpt, F, 1965: Myiasis in man and animals in the old world. Butterworth. 